\title{
CORRIGENDUM
}

\section{A commentary on molecular basis of transfusion dependent beta-thalassemia major patients in Sabah}

\author{
Iswari Setianingsih \\ Journal of Human Genetics (2014) 59, 233; doi:10.1038/jhg.2014.22
}

Correction to: Journal of Human Genetics (2014) 59, 173; doi:10.1038/jhg.2013.142; published online 6 February 2014

After online publication of this article, an error was identified in the list of authors. This error has now been rectified and the corrected article appears in this issue. Iswari Setianingsih is the sole author of this article. 